\title{
NEW CLASSES OF RANDOM TESSELLATIONS ARISING FROM ITERATIVE DIVISION OF CELLS
}

\author{
RICHARD COWAN, * University of Sydney
}

\begin{abstract}
We present new ideas about the type of random tessellation which evolves through successive division of its cells. These ideas are developed in an intuitive way, with many pictures and only a modicum of mathematical formalism - so that the wide application of the ideas is clearly apparent to all readers. A vast number of new tessellation models, with known probability distribution for the volume of the typical cell, follow from the concepts in this paper. There are other interesting models for which results are not presented (or presented only through simulation methods), but these models have illustrative value. A large agenda of further research is opened up by the ideas in this paper.
\end{abstract}

Keywords: Random geometry; tessellation, iterative division; fractal; shape distribution 2000 Mathematics Subject Classification: Primary 60D05; 05B45 Secondary 60G55

\section{Tessellations in $\mathbb{R}^{d}$ with dividing cells}

In the planar case, the class of tessellations that we study can be loosely described as follows. Commence at time $t=0$ with a bounded convex window $W_{0} \subset \mathbb{R}^{2}$, having area 1 and centroid located at the origin. At time $t=1$, we divide $W_{0}$ by a chord using a random mechanism (yet to be defined) and dilate the window and its contents by a linear factor $\sqrt{2}$, using the origin as the dilation centre; the dilated $W_{0}$, now a domain of area 2 , is called $W_{1}$. We select randomly one of the two cells in $W_{1}$ (via methods discussed below). At time $t=2$, we divide the selected cell by our random mechanism applied to that cell and dilate $W_{1}$ and its contents to create a domain $W_{2}$ of area 3. Cell divisions of a selected cell (from the $t$ cells that are extant in $W_{t-1}$ ) continue in this way at the discrete times $t=3,4, \ldots$, with a planar dilation too at each of these epochs. Thus, we create a sequence of increasing domains $W_{3}, W_{4}, \ldots$. The amount of dilation at each stage is chosen to preserve the mean cell area as 1 ; so at each time $t>0$, we dilate by a linear factor of $\sqrt{t+1} / \sqrt{t}$.

Heuristically, this iterative construction should often lead in the limit to a locally finite stationary tessellation of $\mathbb{R}^{2}$ - at least in cases where fractal considerations or other degeneracies do not arise (see later). A similar definition, involving cell division by planes or hyperplanes, applies to higher dimensions.

\section{Some selection and division rules}

We can see that there is a cell selection rule and a cell division rule in the specification of the process. Unless stated otherwise, all random applications of these rules are assumed independent. Regarding cell selection, some possible rules for the planar case are as follows.

Received 2 March 2009; revision received 16 July 2009.

* Postal address: School of Mathematics and Statistics, University of Sydney, NSW 2006, Australia.

Email address: rcowan@mail.usyd.edu.au 
Equally-likely rule. All extant cells have an equal chance of selection.

Area-weighted rule. If the $t$ cells in $W_{t-1}$ under consideration for selection have areas $a_{1}, a_{2}$, $\ldots, a_{t}$, then the chance of cell $j$ being selected is $a_{j} / \sum_{i} a_{i}$ (which equals $a_{j} / t$ ).

Perimeter-weighted rule. If cell $j$ has perimeter $b_{j}$, it is chosen with probability $b_{j} / \sum_{i} b_{i}$.

Corner-weighted rule. If cell $j$ has $c_{j}$ corners, its chance of selection is $c_{j} / \sum_{i} c_{i}$.

The STIT model of Nagel and Weiss [13], named for its stable (ST) properties under a certain type of iteration (IT), employs the perimeter-weighted rule. The evolution of their process is actually in continuous time, with each cell being given an exponentially distributed lifetime whose mean is inversely proportional to the cell's perimeter. Viewed at the division epochs, their process fits into our discrete-time framework - and it is due to this inverse proportionality that the process has our perimeter-weighted selection rule. The properties of the exponential distribution, including the strong Markov feature which implies that residual lifetimes of all cells at any moment in time are exponentially distributed, ensure this.

Although we shall view the models of this paper on a discrete-time scale, with $t$ measuring both division epochs and 'the number of cells minus one', there always exists a convenient continuous-time version based on the appropriate exponentially distributed lifetimes.

Regarding cell division of our polygonal cells, three possible rules are illustrated in Figure 1. These rules lead to a diversity of models and also provide a point of departure for discussing slightly variant ways to divide cells.

The method given in Figure 1(a) will be used most often in the theories we present belowand for this reason we place it first in Figure 1. The method of Figure 1(b) is, however, viewed in the stochastic-geometry literature as the most natural way to construct a random chord. In Figure 1(b), the line $\mathcal{L}$ (and the chord it creates) are called 'uniformly random' (UR). The STIT models use this UR rule.

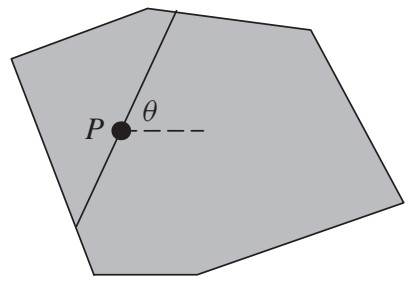

(a)

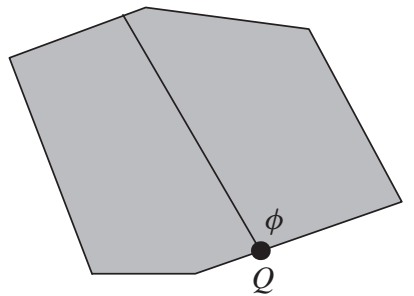

(b)

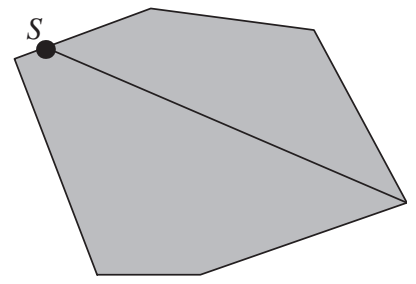

(c)

FIGURE 1: (a) A point $P$ is uniformly distributed within the cell and a chord with slope given by an angle $\theta$, uniformly distributed on $[0, \pi)$, is drawn. (b) A line $\mathcal{L}$ uniformly distributed in the space of lines which hit the cell $\mathcal{C}$ is chosen. The division chord is $\mathcal{L} \cap \mathcal{C}$. A practical implementation of this 'uniformly random' method is: choose a point $Q$ uniformly distributed on the boundary and draw the line at an angle $\phi$ to the boundary-using the probability density function $\frac{1}{2} \sin \phi$ for $\phi$ 's distribution on $[0, \pi)$. (c) Randomly choose a point $S$ on the boundary of the cell, using the uniform distribution, and draw the chord to a randomly chosen 'opposite' corner of the cell. A corner $A$ is 'opposite' $S$ if the chord $S A$ divides the cell into two polygons, each of positive area. If this rule is applied to a triangular starting window $W_{0}$, as in [4], the resulting tessellation has only triangular cells. 


\section{Pictorial representations in $\mathbb{R}^{2}$}

In Figure 2, a square window is divided into 100 cells using some of the selection and division rules described above; see the figure caption. The tessellations we see are of different

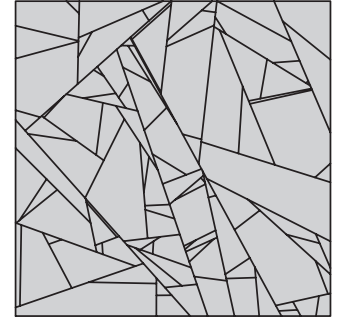

(a)

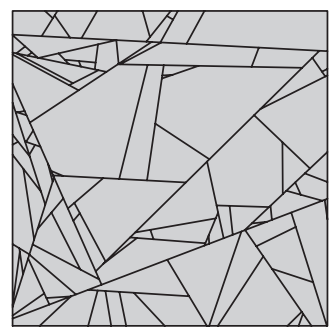

(b)

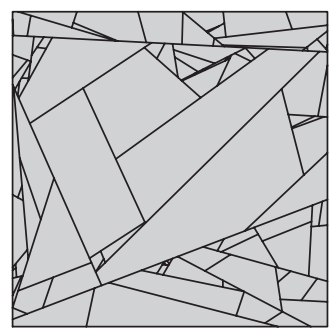

(c)

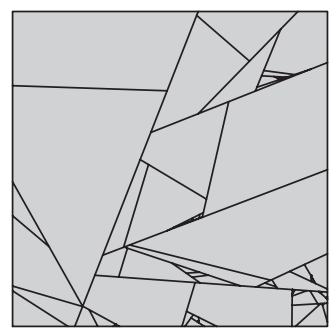

(d)

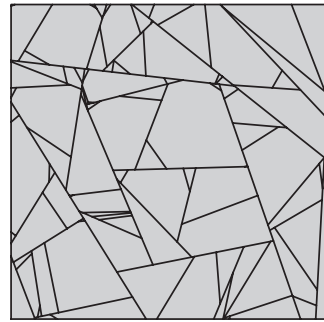

(e)

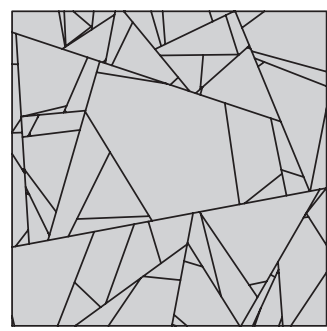

(f)

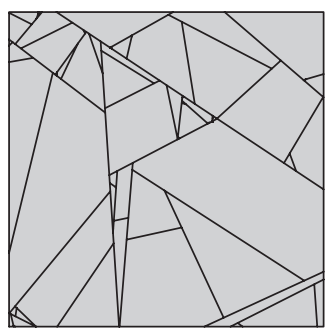

(g)

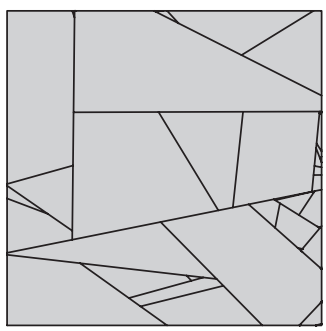

(h)

FIGURE 2: Pictures of $W_{99}$. In (a)-(d), the division rule of Figure 1(a) is applied with (a) area-weighted, (b) perimeter-weighted, (c) corner-weighted, and (d) equally-likely selections. In (e)-(h), the UR rule defined in Figure 1(b) is used-with the selection rule for each case being the same as in the picture directly to the left. Note that, in the corner-weighted and equally-likely cases at the bottom of the figure, many of the 100 cells are too small to be seen. Perhaps the limiting structures here are fractals? At least one suspects that the limiting tessellation 'frame', the union of all edges, is not locally finite. 
character even in these pre-limit pictures at time $t=99$, with greater variability of cell areas as we move from area-weighting at the top to equally likely at the bottom. As for theoretical knowledge of these eight models, little is known, except in case (f). In the limit as $t \rightarrow \infty$, this case corresponds with the STIT model of Nagel and Weiss [13]. The STIT model has some known properties; various mean values and some distributions for certain line segments which appear in the tessellation's 'frame' [12]. Importantly, any property of the typical cell's interior in the STIT model has the same distribution as the same property of a typical cell's interior in the Poisson line process.

Figure 3 shows tessellations of the window with only quadrilateral cells. In Figure 3(a)-(c), all the quadrilaterals are rectangles and, although these are less interesting visually, they offer relatively easy mathematical analysis. The cell division rule in Figure 3(a)-(c) has the dividing line chosen uniformly from the set of all lines which hit the rectangular cell and divide it into two rectangles. This can be viewed as an adaptation of the rule in Figure 1(b), with $\phi$ always equal to $\pi / 2$ (instead of being sampled from the $\frac{1}{2} \sin \phi$ law). Cases (a), (b), and (c) of Figure 3 have differing selection rules (see the figure caption), and these produce tessellations of different character.

Once again, we see greater variability in cell areas as we move from Figure 3(a) to (c). Due to the geometric simplicity of rectangles, we can perform calculations that capture this trend (and we shall briefly present these in the next section).

Planar tessellations comprising only triangular cells arise from the division rule of Figure 1(c), applied to a triangular window $W_{0}$. Tessellations at $t=99$ are shown in Figure 4(a) and (b) for perimeter- and area-weighted selections, respectively. The perimeter-weighted case is developing degenerate (and perhaps fractal-like) qualities even at this early stage of the

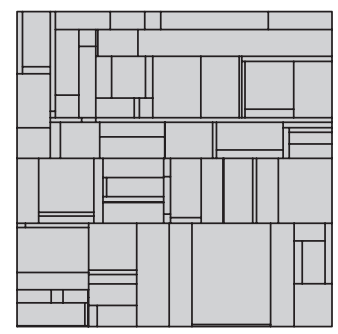

(a)

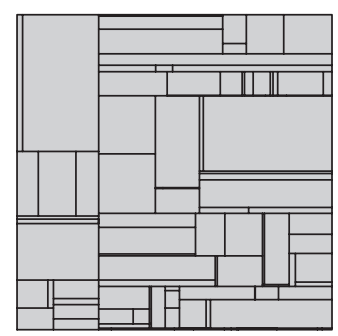

(b)

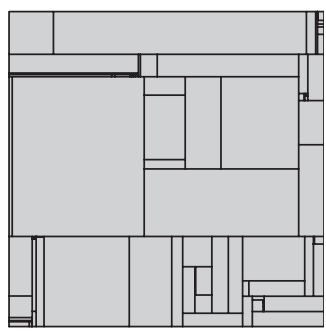

(c)

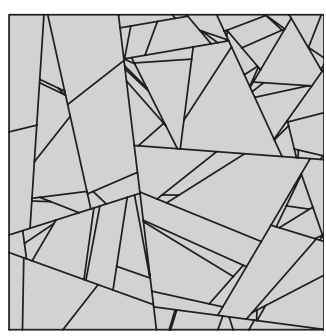

(d)

FIGURE 3: Some tessellations with only quadrilateral cells, these being rectangles in the first three pictures: (a) area-weighted; (b) perimeter-weighted; (c) equally-likely. Note that corner-weighted and equallylikely selection rules are equivalent here. In (d), there is perimeter-weighted selection and a UR division rule censored by the condition that two quadrilateral daughters result. 


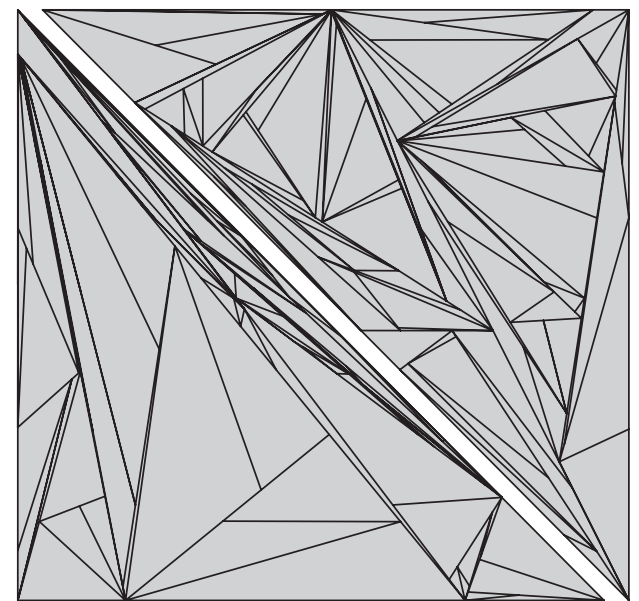

(a)

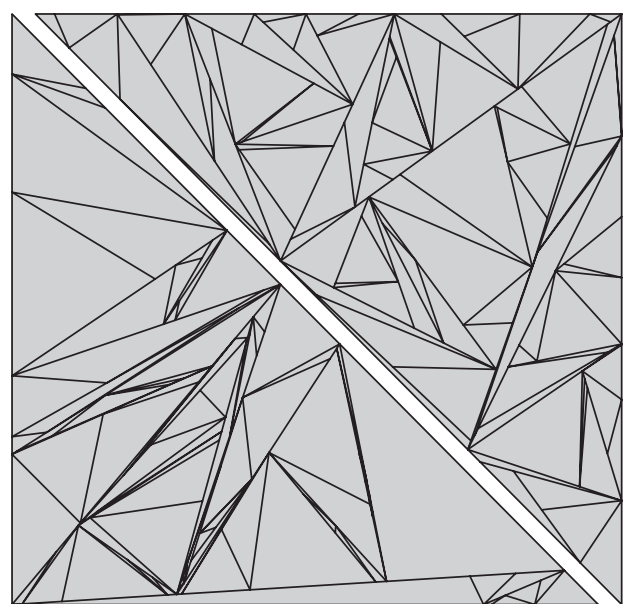

(c)

FIGURE 4: In all four pictures, $t=99$, so the initial triangle $W_{0}$ has been divided into 100 cells. In (a) and (b), a uniformly distributed point on the cell's boundary is joined to the opposite corner: (a) perimeterweighted selection; (b) area-weighted selection. In (c) and (d), a uniformly distributed point on the longest side of the triangular cell is joined to the opposite corner: (c) perimeter-weighted selection; (d) area-weighted selection.

evolution. Perhaps the same can be said about Figure 4(c), where perimeter-weighted selection was used and where the point $S$ was distributed uniformly on the longest side of the triangle (in an attempt to lessen the degenerate tendency). Figure 4(d) also distributes $S$ in this way, although in this case area-weighted selection was used; we see rather well-behaved shapes for the triangular cells, somewhat less elongated than those in Figure 4(b), at least in these early pictures.

\section{Illustrating the trend in cell variability}

In this section we focus on the tessellations comprising only rectangular cells. The structure in Figure 3(b) is the rectangular-celled version of the STIT model. So the area of its typical cell has the same distribution as that for the Poisson line process having only vertical and horizontal lines. Therefore, using the latter model for simplicity of calculation, the typical cell area has a distribution like the product of two independent and identically distributed exponential random variates. Using parameters of the Poisson line process chosen to yield cells of mean area 1, we can write down the distribution of cell areas for this process, and, consequently, for our model of Figure 3(b):

$$
\begin{aligned}
& F(x)_{\text {perim }}=1-2 \sqrt{x} K_{1}(2 \sqrt{x}), \\
& f(x)_{\text {perim }}=K_{0}(2 \sqrt{x})-\frac{1}{\sqrt{x}} K_{1}(2 \sqrt{x})+K_{2}(2 \sqrt{x}),
\end{aligned}
$$

for $x>0$. Here $F$ and $f$ are the distribution and density functions of cell area, and $K_{n}(\cdot)$ is the modified Bessel function (see [1, p. 374]). The subscript 'perim' indicates perimeter-weighted selection; such notation will be used where an emphasis is helpful, but generally omitted in later mathematical calculations where the context is clear. 
We shall prove in Section 6.2 that the typical cell area of the rectangles in Figure 3(a) has the exponential distribution $F(x)_{\text {area }}=1-\mathrm{e}^{-x}, x>0$. So a comparison of the two distributions arising from Figure 3(a)-(b) can be made. Case (b) has variance 3, three times that of (a), whilst the chance that the typical cell has an area greater than 10 is about 130 times higher in (b) than in (a). The chance ratio for areas less than $\frac{1}{10}$ is about 2.45. So there is much more probability mass in the upper and lower tails for case (b).

Regarding the equally-likely case of Figure 3(c), a later analysis (in Section 6.1 and Appendix A) shows degeneracy of the area distribution. When the mean cell area is held at 1 by the dilational scaling, the probability that the typical cell of the Figure 3(c) model has area greater than $x$ tends to 0 for all positive $x$; the occasional huge cell is insignificant in number compared with the number of cells which have vanishingly small area. Cell areas have infinite variance in these cases.

The models of Figure 4(a) and (c) have a similar look to that of the Figure 3(c) model, appearing to converge to a tessellation with the typical cell having area 0 with probability 1 .

\section{Geometry-independent apportionment of volume}

In $\mathbb{R}^{2}$, each of the chords can be given a direction assigned by coin toss. The two daughter cells can now be classified as 'left' or 'right' of the directed chord which divided their mother. The two daughters have identical statistical properties because a chord having a certain direction is as likely as the same chord with the opposite direction. A similar partition of polytopes in $\mathbb{R}^{d}, d>2$, also yields two identically distributed daughter cells: one in the left half-space, the other in the right.

Consider now a division rule in $\mathbb{R}^{d}$ that, when applied at time $t$, apportions the volume of the mother cell between her two daughters in a manner which is independent of all geometric features within $W_{t-1}$-except (perhaps) the volume of the mother cell.

Division rules with this geometry-independent apportionment (GIA) have the following structure. In $\mathbb{R}^{d}$, division creates left daughters whose volume is a proportion $U$ of the mother cell, where $U$ has the distribution function $G(\cdot \mid v)$, say, on $[0,1]$. Here, $v$ is the volume of the mother (though in most examples this conditioning argument will be dropped because $U$ is independent of the mother's volume). The distribution must be symmetric, that is, $G(x \mid v)=1-\lim _{u \uparrow(1-x)} G(u \mid v)$, and we shall apply the regularity condition that $U \in(0,1)$, so $G(0 \mid v)=0$ and $\lim _{x \uparrow 1} G(x \mid v)=1$.

Consider the triangular-celled model in $\mathbb{R}^{2}$, which is illustrated and defined in Figure 5. It is clear that the chord drawn at each time $t>0$ has geometry-independent isotropy (GII) (that is, the chord has uniformly distributed orientation on $[0, \pi)$ independently of the geometry within $\left.W_{t-1}\right)$. But does it have the GIA property?

Suppose that the corner which $\mathcal{L}$ hits has an internal angle $\psi$. This angle is split uniformly by the GII chord that $\mathcal{L}$ defines, but the area apportionment is more complicated. Calculations show that area apportionment $U$ has conditional probability density function, for $0<u<1$, as follows:

$$
\begin{aligned}
g(u \mid \psi, r)=\frac{1}{2 \psi}( & \frac{r \sin \psi}{r^{2}(1-u)^{2}+2 r u \cos \psi(1-u)+u^{2}} \\
& \left.+\frac{r \sin \psi}{r^{2} u^{2}+2 r u \cos \psi(1-u)+(1-u)^{2}}\right),
\end{aligned}
$$

where $r$ is the relative length of the two sides (shorter/longer) which subtend $\psi$. So, clearly, there is geometry dependence in the area apportionment; therefore, the model is not GIA. 


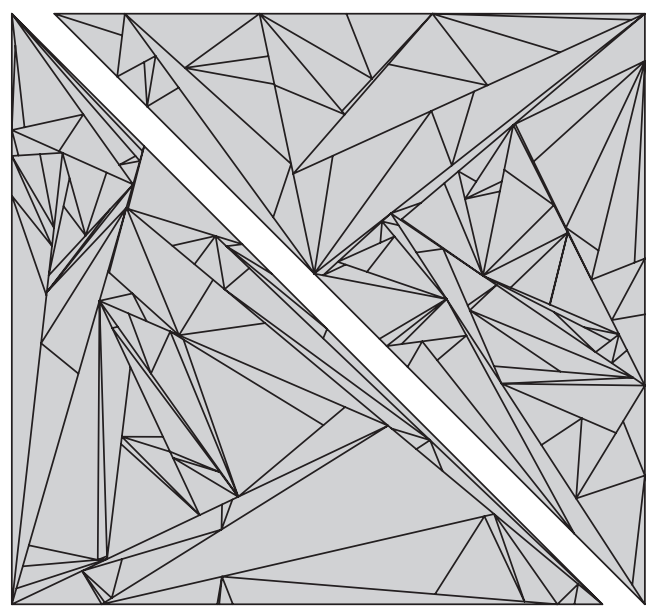

(a)

(b)

FIGURE 5: Triangles constructed using 'geometry-independent isotropic' chords. We sample an angle uniformly distributed on $[0, \pi)$ and use the unique line $\mathcal{L}$ having this angle as slope and dividing the triangular cell $C$ into two triangles. The dividing chord is $\mathcal{L} \cap C$. The two cases are: (a) perimeterweighted selection; (b) area-weighted selection.

Are there models which have GIA? Yes; we have encountered some already. Those illustrated in Figure 3(a)-(c) are applicable; they have $U$ distributed uniformly on $(0,1)$. Likewise, all the models in Figure 4.

But most importantly, the division rule introduced in Figure 1(a) has GIA-and obviously GII too. So all models which employ the rule of Figure 1(a) provide further examples of this area apportionment that is independent from the geometry. We shall prove this fact in the next two sections.

\section{Mapping into a process on an interval in $\mathbb{R}$}

Consider the unit interval $I_{0}:=\left[-\frac{1}{2}, \frac{1}{2}\right]$ in $\mathbb{R}$. It has length measure of 1 , just as $W_{0} \subset \mathbb{R}^{d}$ has volume measure of 1 . When a hyperplane (or chord/plane if $d \leq 3$ ) divides $W_{0}$, yielding a left daughter having volume $v$, we place a point in $I_{0}$ dividing this interval in the same left-toright proportion $v: 1-v$. Thus, a link is created between the left daughter of $W_{0}$ and the left daughter of $I_{0}$, and likewise between the right daughters. We dilate both $W_{0}$ and $I_{0}$, so that the mean cell measure in both situations is 1 . The interval $I_{0}$ becomes $I_{1}:=[-1,1]$ at time $t=1$ post dilation, whilst $W_{0}$ becomes $W_{1}$.

When the second cell-dividing hyperplane is placed in $W_{1}$, we also place a point in $I_{1}$ dividing the linked interval in the same left-to-right proportions given by the division event in $d$ dimensions. We continue in this way, with a linkage established between the cells in $W_{t} \subset \mathbb{R}^{d}$ at time $t$ and the subintervals of $I_{t}:=[-(t+1) / 2,(t+1) / 2] \subset \mathbb{R}$.

Whilst much of the geometric information of the $d$-dimensional tessellation is lost in this 'mapping', the volume of a $d$-dimensional cell is measured by the length of its linked onedimensional interval. This is an important idea, with some startling consequences.

In particular, GIA constructions in $\mathbb{R}^{d}$ which use either a volume-weighted or equally-likely selection rule can be analysed using the one-dimensional linkage. For example, if volume weighting is employed in cell selection (and GIA applies), this situation can be emulated within the one-dimensional analogue by using length-weighted interval selection. 
So our mathematical analysis becomes one-dimensional, focusing on the windows $I_{0}, I_{1}$, $I_{2}, \ldots \subset \mathbb{R}$. We find that a simple equation links the distribution function $L_{t}$ of the left daughter's length (after the cell division and dilation which occurs at time $t$ ) with the distribution function $S_{t-1}$ of length for the cell selected (from those in $I_{t-1}$ ) to be the mother:

$$
L_{t}(x)=S_{t-1}\left(\frac{t x}{t+1}\right)+\int_{v=t x /(t+1)}^{v=t} G\left(\frac{t x}{(t+1) v} \mid v\right) \mathrm{d} S_{t-1}(v), \quad t \geq 1 .
$$

The same distribution function describes the length of the right daughter.

When the selection rule is perimeter-weighted or corner-weighted, an analysis using only the one-dimensional linkage is not possible, but some considerable progress can be made in the volume-weighted and equally-likely cases. We explore these two cases now.

\subsection{Equally-likely selection}

In the case where the equally-likely selection rule is applied, $S_{t-1}$ takes a trivial form, namely $S_{t-1}=F_{t-1}$, where $F_{t}(x)$-written more formally as $F_{t}(x)_{\text {equal }}$ in our feature results-denotes the probability that a typical segment of $I_{t}$ has length less than or equal to $x$. Here a typical segment is one randomly chosen from those extant. Also, it is easy to see that $\bar{S}_{t-1}$, defined as the distribution function for the length of a cell given that it is not selected, equals $F_{t-1}$ too. We are then able to relate $F_{t}$ to $F_{t-1}$. Clearly, $F_{t}(x)=0$ for $x \leq 0$ and $F_{t}(x)=1$ for $x \geq(t+1)$, whilst $F_{0}(x)=0$ for $0<x<1$. For $0<x<(t+1)$ and $t \geq 1$,

$$
\begin{aligned}
F_{t}(x)=\frac{1}{t+1} & {\left[2 L_{t}(x)+(t-1) \bar{S}_{t-1}\left(\frac{t x}{t+1}\right)\right] } \\
=\frac{1}{t+1} & {\left[2 F_{t-1}\left(\frac{t x}{t+1}\right)+2 \int_{v=t x /(t+1)}^{v=t} G\left(\frac{t x}{(t+1) v} \mid v\right) \mathrm{d} F_{t-1}(v)\right.} \\
& \left.\quad+(t-1) F_{t-1}\left(\frac{t x}{t+1}\right)\right] \\
= & F_{t-1}\left(\frac{t x}{t+1}\right)+\frac{2}{t+1} \int_{v=t x /(t+1)}^{v=t} G\left(\frac{t x}{(t+1) v} \mid v\right) \mathrm{d} F_{t-1}(v) .
\end{aligned}
$$

Theorem 1. When $G(\cdot \mid v)$ does not depend on $v$, (2) has a unique solution given by

$$
F_{t}(x)_{\text {equal }}=\sum_{n=1}^{t} p_{n}(t) G_{n}\left(\frac{x}{t+1}\right), \quad t \geq 1,0<x<t+1,
$$

where $p_{n}(t)$ is the coefficient of $z^{n}$ in $2 z \Gamma(2 z+t) /(t+1) ! \Gamma(2 z+1)$, which equals $(2 z / t(t+$ 1)) $\prod_{i=1}^{t-1}(1+2 z / i)$ when $t>1$, and $G_{n}$ is calculated as follows:

$$
\begin{gathered}
G_{1}(x)=G(x), \\
G_{n}(x)=G_{n-1}(x)+\int_{v=x}^{v=1} G\left(\frac{x}{v}\right) \mathrm{d} G_{n-1}(v), \quad n \geq 2 .
\end{gathered}
$$

Corollary 1. Let $V_{t}$ be the length of a typical cell extant at time $t$, that is, a cell randomly selected from the cells of $I_{t}$. Then $\operatorname{var}\left(V_{t}\right)=(t+1)^{2} \sum_{n=1}^{t} p_{n}(t)\left(\mathrm{E} U^{2}\right)^{n}-1 \rightarrow \infty$ as $t \rightarrow \infty$. 
Proofs of Theorem 1 and Corollary 1 are given in Appendix A, as the necessary detail involved detracts from the broad themes we are outlining.

One example where the sequence of functions $G_{n}, n \geq 1$, defined by (4), can be explicitly calculated is known from the early work of Halmos [6] on random splitting of the unit interval. This is the case where $U$ is uniformly distributed on $[0,1]$; then $G_{n}$ and its density $g_{n}$ are as follows. For $0<x<1$,

$$
G_{n}(x)=x \sum_{j=0}^{n-1} \frac{(-\log x)^{j}}{j !}, \quad g_{n}(x)=\frac{(-\log x)^{n-1}}{(n-1) !} .
$$

So, in this case we can easily write $F_{t}$, or better still the density $f_{t}$, as

$$
f_{t}(x)_{\text {equal }}=\sum_{n=1}^{t} \frac{p_{n}(t)(\log (t+1)-\log x)^{n-1}}{(t+1)(n-1) !}, \quad 0<x<t+1 .
$$

A graph of the density function is plotted in Figure 6(a). Another example is illustrated in Figure 6(b), this being the division rule least likely to create the abundance of small cells seen in other cases. Here a selected cell is always divided into two daughters of equal area.

In Figure 6(b), the distribution function $F_{t}$ is plotted for $0<x<1$ and various values of $t$. At small $x$, we note that $F_{200}(x)>F_{75}(x)>F_{30}(x)>F_{10}(x)$. This, combined with the infinite variance finding in Corollary 1 , suggests that segment lengths (cell volumes) are becoming concentrated at 0 . We now show this formally.

Theorem 2. When the selected cell is always divided into two daughters of equal volume, $F_{t}(x)_{\text {equal }} \rightarrow 1$ for all $x \geq 0$ as $t \rightarrow \infty$.

Theorem 2, which is proved in Appendix A, suggests intuitively that the equally-likely selection rule leads to structures which are degenerate in the sense of the theorem. We have not proved this style of degeneracy for all $G$, but it is easy to show that $\operatorname{var}\left(V_{t}\right)$ is minimal when
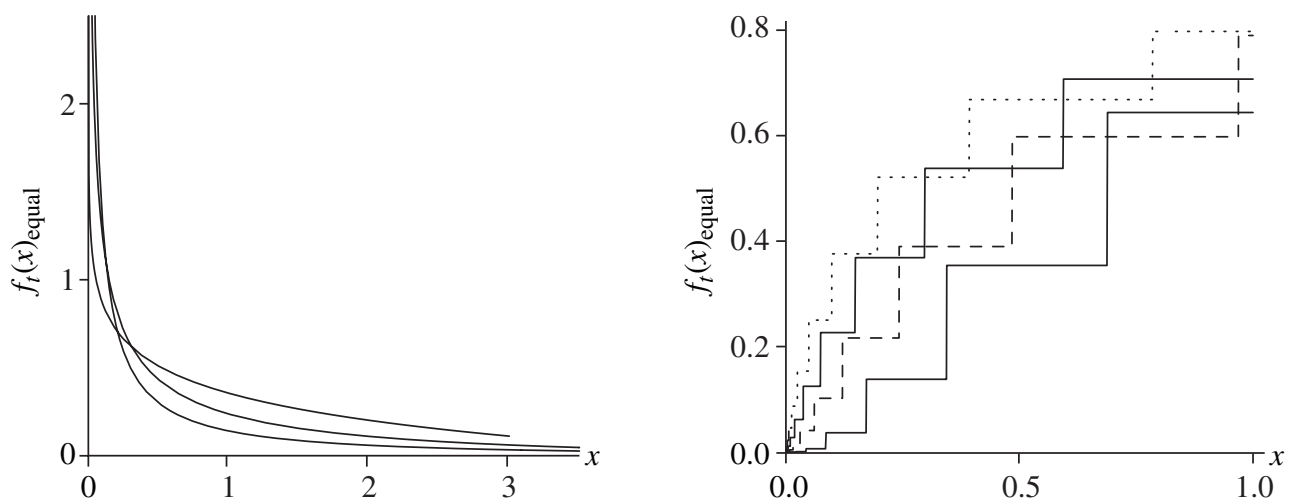

FIGURE 6: (a) The probability density function $f_{t}$ (shown in (5)) when $U$ is distributed uniformly on $(0,1)$ for $t=2,5,20$. The three curves are identified by the ordering $\left.f_{2}(1)>f_{5}(1)>f_{20}(1)\right)$. (b) The distribution function $F_{t}$ for $t=10$ (lower solid line), $t=30$ (dashed line), $t=75$ (upper solid line) and $t=200$ (dotted line) in the case where selected segments are always divided exactly in half-that is, when $G(x)=0, x<\frac{1}{2}$, and $G(x)=1, x \geq \frac{1}{2}$. 
cells split in the manner of Theorem 2 (see the proof of Corollary 1 in Appendix A). So the rule of Theorem 2 has the 'least degenerate' $G$ in at least one sense.

Further work is needed to establish if the limiting structure has fractal dimension and, if so, what the dimension is.

\subsection{Volume-weighted selection}

In the case where a volume-weighted selection rule is applied, results in $\mathbb{R}^{d}$ can be captured by an analysis of the length-weighted process in $\mathbb{R}$. Using standard ideas concerning lengthweighted sampling (and a notation where $F_{t}(x)_{\text {volume }}$ is implied whenever $F_{t}(x)$ is used), we see that $S_{t-1}(x)=\int_{u \leq x} u \mathrm{~d} F_{t-1}(u)=x F_{t-1}(x)-\int_{0}^{x} F_{t-1}(u) \mathrm{d} u$. (Readers will be more familiar with this assertion in the case where the distribution functions have densities $s$ and $f$-then $s_{t-1}(x)=x f_{t-1}(x) / \mathrm{E}$ (typical cell length), and here the typical cell has mean length equal to 1 .)

Because $F_{t-1}=\left[S_{t-1}+(t-1) \bar{S}_{t-1}\right] / t$, we have $\bar{S}_{t-1}=\left[t F_{t-1}-S_{t-1}\right] /(t-1)$. Now, by applying (1), the relationship between $F_{t}$ and $F_{t-1}$ is, for $0<x<(t+1)$,

$$
\begin{aligned}
F_{t}(x)=\frac{1}{t+1} & {\left[2 L_{t}(x)+(t-1) \bar{S}_{t-1}\left(\frac{t x}{t+1}\right)\right] } \\
=\frac{1}{t+1} & {\left[2 S_{t-1}\left(\frac{t x}{t+1}\right)+2 \int_{v=t x /(t+1)}^{v=t} G\left(\frac{t x}{(t+1) v} \mid v\right) \mathrm{d} S_{t-1}(v)\right.} \\
& \left.+t F_{t-1}\left(\frac{t x}{t+1}\right)-S_{t-1}\left(\frac{t x}{t+1}\right)\right] \\
=\frac{1}{t+1} & {\left[t\left(1+\frac{x}{t+1}\right) F_{t-1}\left(\frac{t x}{t+1}\right)-\int_{0}^{t x /(t+1)} F_{t-1}(u) \mathrm{d} u\right.} \\
& \left.+2 \int_{v=t x /(t+1)}^{v=t} G\left(\frac{t x}{(t+1) v} \mid v\right) v \mathrm{~d} F_{t-1}(v)\right]
\end{aligned}
$$

For example, if $U$ is uniformly distributed on $[0,1]$ then $G(x)=x, 0 \leq x \leq 1$, and so

$$
\begin{aligned}
F_{t}(x)=\frac{1}{t+1}[ & t\left(1+\frac{x}{t+1}\right) F_{t-1}\left(\frac{t x}{t+1}\right)-\int_{0}^{t x /(t+1)} F_{t-1}(u) \mathrm{d} u \\
& \left.+\frac{2 t x}{t+1}\left(1-F_{t-1}\left(\frac{t x}{t+1}\right)\right)\right] \\
=\frac{1}{t+1} & {\left[t\left(1-\frac{x}{t+1}\right) F_{t-1}\left(\frac{t x}{t+1}\right)-\int_{0}^{t x /(t+1)} F_{t-1}(u) \mathrm{d} u+\frac{2 t x}{t+1}\right] . }
\end{aligned}
$$

We actually know a great deal about this one-dimensional partitioning and rescaling of the interval $I_{0}$ without getting too involved with (7), because the combination of (a) the lengthweighted selection of a subinterval of $I_{t}$ and (b) the uniform proportion splits of the chosen subinterval can both be achieved by simply sampling a uniformly random point in $I_{t}$. So the one-dimensional process is just the sequential accumulation of uniformly distributed points $P_{1}, P_{2}, P_{3}, \ldots$ in $I_{0}$, with progressive dilation to preserve the mean interval length.

It is well known that this collection of points converges in distribution to the stationary Poisson point process on $\mathbb{R}$ of intensity 1 , and the typical interval length in the limit has the exponential distribution $1-\mathrm{e}^{-x}, x>0$. Immediately, we can state the following theorem. 
Theorem 3. In any of our iterative tessellations of $\mathbb{R}^{d}$ using volume-weighted selection and a division rule which yields daughter volumes as a uniformly distributed proportion of mother volumes, typical cell volumes converge in distribution as $t \rightarrow \infty$ to a random variable having $F(x)_{\text {volume }}=1-\mathrm{e}^{-x}, x>0$.

Proof. The discussion above will be proof enough for most readers, but those unfamiliar with this type of convergence to a Poisson process can prove the theorem by solving (7) for finite $t$. The unique solution for all $t \geq 0$ is

$$
F_{t}(x)_{\text {volume }}= \begin{cases}0, & x \leq 0, \\ 1-\left(1-\frac{x}{t+1}\right)^{t}, & 0<x<t+1, \\ 1, & x \geq(t+1) .\end{cases}
$$

Substitution into (7) will prove this. The genesis of the exponential limit as $t \rightarrow \infty$ is therefore clear.

Corollary 2. The $\mathbb{R}^{2}$ examples shown in Figures 3(a), 4(b), 4(d), and, most importantly, 2(a), have exponentially distributed cell areas.

The first three examples mentioned in Corollary 2 obviously comply with the conditions of Theorem 3, as do many other area-weighted tessellation models. For example, we can vary the division rule in the rectangular model of Figure 3(a) by censoring the mechanism to only allow chords which hit the longer side of a rectangular cell (or on a random side if the rectangle is a square); see Figure 7(a). This division rule still apportions area uniformly, as it does in the other examples captured in Figure 7 (see the figure caption).

The conformity of the most important example in Corollary 2-that based on Figure 2(a) with the conditions of Theorem 3 is less obvious. Naturally, it depends on the division rule from Figure 1(a) creating daughter cells whose area is a uniformly distributed proportion of their mother cell's area. We now discuss this division rule (or a generalised version of it) in greater detail.

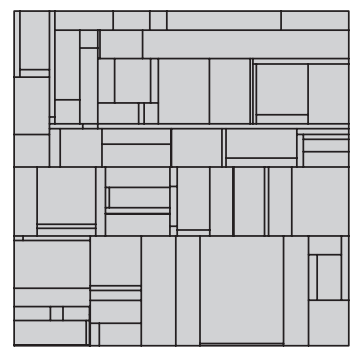

(a)

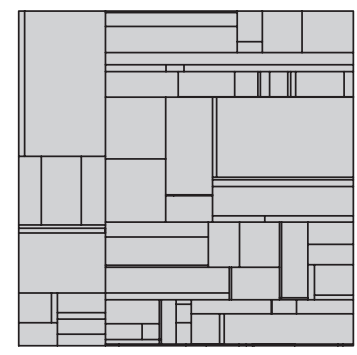

(b)

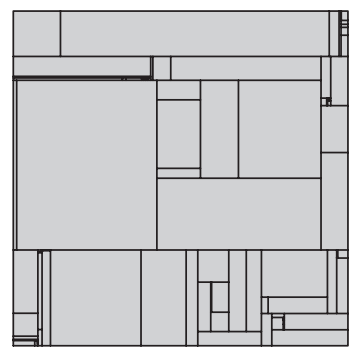

(c)

FIGURE 7: Using area-weighted selection in all three cases, we see the effects of different division rules. (a) The chord is uniformly distributed from those that orthogonally hit the long side of the cell selected for division. (b) A point $P$ is placed uniformly in the selected cell and the direction of the chord, horizontal or vertical, is decided by tossing a balanced coin. (c) The chord is uniformly distributed from those that orthogonally hit the chord which divided the mother of the selected cell. So sister cells will have a geometric dependency: parallel dividing chords. Despite this dependency, the area apportionments of the two sisters are independent random variables. 


\section{Division rules generalising that of Figure 1(a)}

Let us firstly generalise the rule by allowing the angle $\theta$ to be chosen by any method, random or not, geometry independent or not, isotropic or not. Then, after $\theta$ has been chosen, the point $P$ is placed uniformly in the cell—an action obviously independent of $\theta$. This $(P, \theta)$ pair defines the chord.

Theorem 4. If this $(P, \theta)$ construction is applied to any mother cell in $\mathbb{R}^{2}$, the left daughter has an area which, proportional to that of its mother, is uniformly distributed on $(0,1)$. The right daughter has the same distribution. A similar statement holds in $\mathbb{R}^{d}$ for volumes, where the angle $\theta$ is replaced by the orientation specifications of an arbitrary hyperplane-and areas are replaced by volumes.

Proof. For simplicity, let us say that the mother cell $M \subset \mathbb{R}^{d}$ is a connected set (as in all of our examples to date). Consider the projection of $M$ onto a line orthogonal to hyperplanes of the chosen orientation (or, in $\mathbb{R}^{2}$, orthogonal to lines having slope $\theta$ ). The projection, a line segment, is labelled $L R$ (i.e. left end, right end) and the projection of the random point $P$ is labelled $P_{\perp}$. Let $X$ be the random length $L P_{\perp}$, and define $H(x):=\mathrm{P}\{X \leq x\}$. Because $P$ is uniformly distributed in $M, H(x)=V(x) / V$, where $V$ is the volume of $M$ and $V(x)$ is its volume to the left of a hyperplane through $P$ of the chosen orientation. Note that $H$ is a continuous function; this means that the random variable $H(X)$ is distributed uniformly on $[0,1]$. But $H(X)=V(X) / V$, so $V(X) / V \sim \operatorname{Unif}(0,1)$.

It is important to note that the orientation of the dividing plane (line) can be arbitrarily decided. This opens up a vast repertoire of tessellation models more complicated than that illustrated in Figure 2(a) — and, for each such model, the cell volume (area) is exponentially distributed. Using planar examples for simplicity, we can have a dependence of $\theta_{t}$ (the slope for the cell division at epoch $t$ ) on $\theta_{t-1}, \theta_{t-2}, \ldots$, say, or on various geometric features of the tessellation constructed up to time $t-1$, and still have exponentially distributed areas. For example, the slope $\theta$ for a given cell might be chosen to be at a random angle $\kappa$ to the line which divided that cell from its sister, where (say) $\kappa$ is distributed with mean $\pi / 2$ and some small variance. (Biological cells in two-dimensional epithelia have a tendency to divide in this way, roughly orthogonal to their mother cell's dividing line-exact orthogonality resulting in the picture seen already in Figure 7(c).) Or the slope $\theta$ for a chosen cell might equal the slope of that cell's sister if the sister has already divided; otherwise, it is sampled randomly.

\section{Other considerations}

We started this paper with many different selection rules and division rules, but our theoryafter introducing the concept of GIA and showing via Theorems 1 and 2 that equally-likely selection rules lead to degenerate tessellations-has narrowed its focus to just one selection rule, namely volume-weighted selection. In this context, Theorems 3 and 4 become the major findings of this paper. These theorems are simply proved, but it is their recognition, and the usage that they have in combination, which is profound. They provide explicit volume distributions for an enormous class of tessellation models, and the amazingly simple exponential distribution is the common form in most cases that we have discussed.

Along the way, the reader will have seen slight variations on the theme and will undoubtedly have imagined many more. There are also many theoretical issues not yet discussed.

In the remainder of this paper we discuss four of these issues. The first takes us to a selection rule which lies beyond those that have guided our paper to date. It demonstrates that many of 
the ideas extend to quite complicated selection rules. The second issue involves a departure from uniformly distributed volume apportionment. An example is explicitly solved and this provides hope that the methods can be applied fairly generally. The third issue is about cell shape; it will already have been noted that some pictures have rather rotund shapes whilst others have many very elongated shapes. It would be worthwhile to establish if, in the context (say) where cell volumes converge in distribution to the exponential law, there is a stable distribution of shapes, or if shapes become degenerate as $t \rightarrow \infty$. The fourth issue is the convergence of our iterative cell division process. The increasing windows $W_{0}, W_{1}, W_{2}, \ldots$ cover $\mathbb{R}^{d}$ in the limit, but does the structure within converge to a locally finite stationary tessellation, or can it sometimes be a fractal? We do not resolve this question in the current paper, but some comment is given.

\subsection{Selecting the cell with the largest volume}

The linkage between cells of $W_{t} \subset \mathbb{R}^{d}$ and segments on the interval $I_{t}$, discussed in Section 6 , can be exploited in any situation where the properties of the segments are able to be analysed. To illustrate this, we consider models where we always select the cell having the largest volume (dividing it in a manner so that the ratio of left daughter to mother volumes is uniformly distributed). The segments formed in the one-dimensional setting can be analysed; experimentation using simulation leads to the conjecture of a very simple result for segment lengths.

Using the GII and GIA division rules of Figure 1(a), we draw in Figure 8(a) a typical planar realisation of the model at the stage where the window is partitioned into 200 cells. The picture seems very 'well behaved', with no cells of extremely large or extremely small area.

A simulation of the equivalent segmentation of $I_{0}$ can be conducted very easily. The results of this at the stage where 50000 segments exist yield the rather simple histogram of segment length shown in Figure 8(b). It appears that the distribution of cell areas is converging to the Unif[0, 2] law.

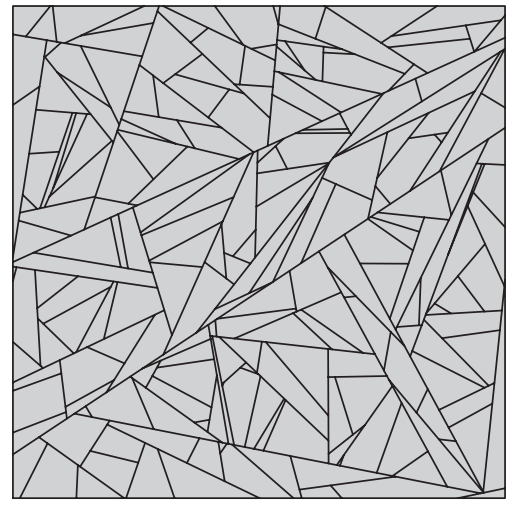

(a)

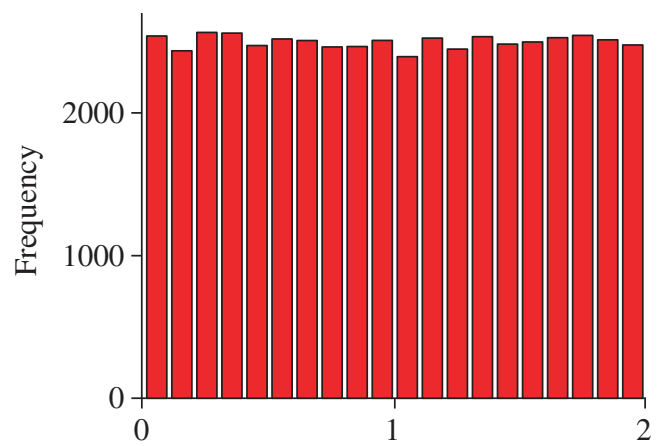

(b)

Figure 8: (a) The division rule is that of Figure 1(a), but now the cell selected is the one with largest area. (b) A histogram of segment lengths arising from segmentation of the unit interval $I_{0}$ into 50000 segments, dilation being applied progressively after dividing the longest segment uniformly at each stage. Only those segments of length less than or equal to 2 are displayed; there were 35 segments greater than 2 , the sample maximum being 2.00101 . 
Conjecture 1. If $I_{0}:=\left[-\frac{1}{2}, \frac{1}{2}\right]$ is segmented by the sequential placement of points, each being uniformly distributed in the longest segment from those extant at each stage, then the typical segment length (scaled to keep its mean as 1) converges in distribution to the Unif[0, 2] law.

This conjecture has not yet been proved, but, if it is true then the typical cell area seen in Figure 8(a) also converges to this distribution. A similar result applies to volumes in higherdimensional tessellations of this type, as it does whenever the linkage ideas hold.

\subsection{Volume-weighted selection with $\boldsymbol{U}$ not uniform}

We have shown that (6) has a simple solution when $U$ is uniformly distributed. A solution of (6) may also be possible in other cases and in this subsection we provide it when $U$ has a simple symmetric beta distribution whose probability density function is $g(u):=6 u(1-u)$, $0<u<1$. The derivation details, which are very lengthy, are omitted.

Planar tessellations that apportion area in this way are easy to construct; Figure 9(a) shows two cases, one with rectangular cells that divide with a line orthogonal to the longer side (and apportionment governed by $g$ ) and another with triangular cells and a division line from a $g$-distributed point on the longest side to the opposite corner.

We can show that the solution of (6) for finite $t$ takes the following form using this $g$, that is, using $G(u):=u^{2}(3-2 u), 0<u<1$. For all $t \geq 1$ and $0 \leq x \leq t+1$,

$$
F_{t}(x)_{\text {volume }}=\left(A(t) x^{3}+B(t) x^{4}+C(t) x^{5}\right) \log \left(\frac{x}{t+1}\right)+\sum_{n=2}^{t+1} a_{n}(t) x^{n},
$$

where

$$
\begin{aligned}
A(t) & :=\frac{2 t(t-1)\left(2-5 t+5 t^{2}\right)}{(t+1)^{4}}, & B(t) & :=\frac{15 t(t-1)^{3}(t-2)}{2(t+1)^{5}}, \\
C(t) & :=\frac{t(t-1)^{2}(t-2)^{2}(t-3)}{(t+1)^{6}}, & a_{2}(t) & :=\frac{t\left(5 t^{2}+1\right)}{(t+1)^{3}},
\end{aligned}
$$

these entities converging to finite limits as $t \rightarrow \infty$. Some coefficients take divergent forms, namely

$$
\begin{aligned}
& a_{3}(t):=\frac{12-68 t+139 t^{2}-100 t^{3}+5 t^{4}}{3(t+1)^{4}}+A(t) \sum_{n=1}^{t} \frac{1}{n}, \\
& a_{4}(t):=\frac{3(t-1)\left(40-122 t+61 t^{2}+60 t^{3}-35 t^{4}\right)}{8(t+1)^{5}}+B(t) \sum_{n=1}^{t} \frac{1}{n}, \\
& a_{5}(t):=\frac{(t-1)(t-2)\left(90-66 t-250 t^{2}+243 t^{3}-53 t^{4}\right)}{15(t+1)^{6}}+C(t) \sum_{n=1}^{t} \frac{1}{n},
\end{aligned}
$$

whilst, for $n \geq 6$, we have the set of convergent coefficients

$$
a_{n}(t):=\left(\begin{array}{c}
t \\
n-1
\end{array}\right) \frac{12(-1)^{n+1}(n-1)\left(5-4 n+n^{2}+10 t-5 n t+5 t^{2}\right)}{(t+1)^{n+1} n(n-3)(n-4)(n-5)} .
$$

From (8), it is easy to establish the limiting distribution $F$ as $t \rightarrow \infty$. We find that, as $t \rightarrow \infty$,

$$
a_{n}(t) \rightarrow \frac{60(-1)^{n+1}}{n(n-2) !(n-3)(n-4)(n-5)}, \quad n \geq 6,
$$



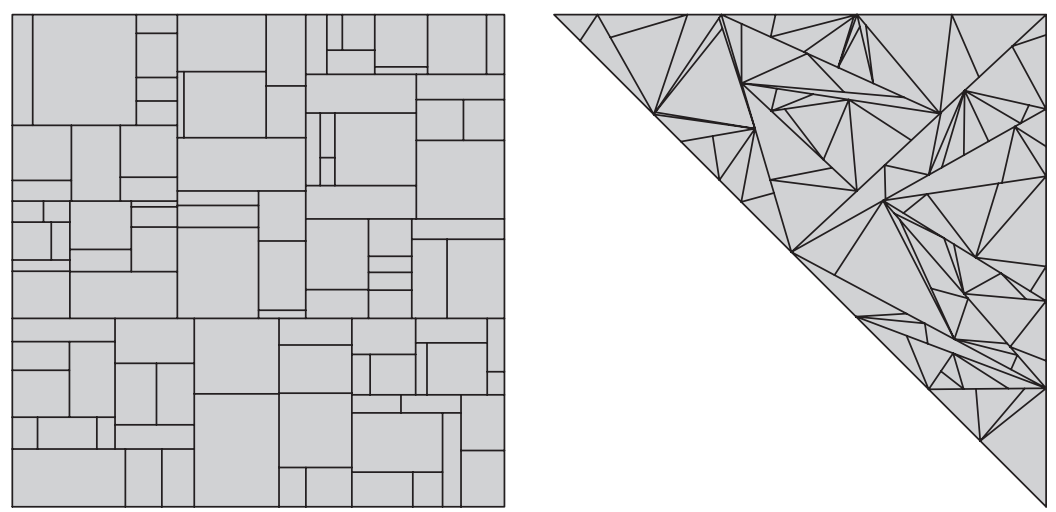

(a)

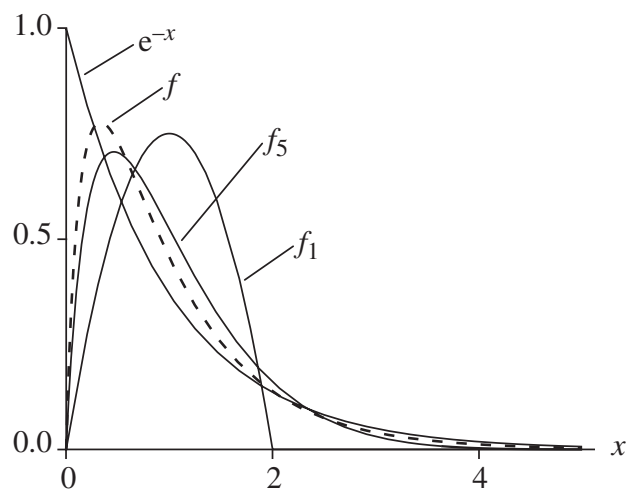

(b)

FIGURE 9: (a) In both tessellations, a point is randomly placed on the longer (or longest) side of the selected cell according to the distribution $G$ whose density is $g(u)=6 u(1-u), 0<u<1$. In the case of rectangular cells, the dividing line passes through this point, orthogonal to the long side. In the triangular case, the chord passes from the random point to the opposite corner. (b) The dashed curve shows the function $f$, as defined in (9), together with $f_{1}, f_{5}$, and $\mathrm{e}^{-x}$. The area of typical cells in both pictures are distributed with probability density $f$, in the limit.

and

$$
\begin{aligned}
\sum_{n=6}^{t+1} a_{n}(t) x^{n} \rightarrow & \frac{120-600 x^{2}-200 x^{3}+1575 x^{4}+424 x^{5}}{120} \\
& -\frac{\mathrm{e}^{-x}}{2}\left(2+2 x-9 x^{2}-13 x^{3}-2 x^{4}\right) \\
& -\frac{x^{3}}{2}\left(20+15 x+2 x^{2}\right)\left(\gamma+\log x+\int_{x}^{\infty} \frac{\mathrm{e}^{-v}}{v} \mathrm{~d} v\right),
\end{aligned}
$$

where $\gamma$ is Euler's constant. Obviously, $A(t) \rightarrow 10, B(t) \rightarrow \frac{15}{2}, C(t) \rightarrow 1$, and $a_{2}(t) \rightarrow 5$. These facts combined with the known convergence of $\left(\sum_{1}^{t+1} 1 / n-\log (t+1)\right)$ to $\gamma$, which 
implies that $\left(\sum_{1}^{t} 1 / n-\log (t+1)\right)$ also converges to $\gamma$, yields

$$
\begin{gathered}
a_{3}(t)-A(t) \log (t+1) \rightarrow 10 \gamma+\frac{5}{3}, \quad a_{4}(t)-B(t) \log (t+1) \rightarrow \frac{15}{2} \gamma-\frac{105}{8}, \\
a_{5}(t)-C(t) \log (t+1) \rightarrow \gamma-\frac{53}{15} .
\end{gathered}
$$

This leads to an interesting limiting distribution and corresponding density function:

$$
\begin{aligned}
& F(x)_{\text {volume }}=1-\frac{\mathrm{e}^{-x}}{2}\left(2+2 x-9 x^{2}-13 x^{3}-2 x^{4}\right)-\frac{x^{3}}{2}\left(20+15 x+2 x^{2}\right) \int_{x}^{\infty} \frac{\mathrm{e}^{-v}}{v} \mathrm{~d} v \\
& f(x)_{\text {volume }}=5 x \mathrm{e}^{-x}\left(2+5 x+x^{2}\right)-5 x^{2}\left(6+6 x+x^{2}\right) \int_{x}^{\infty} \frac{\mathrm{e}^{-v}}{v} \mathrm{~d} v
\end{aligned}
$$

The function $f$ is the dashed curve plotted in Figure 9(b) alongside the exponential density $\mathrm{e}^{-x}, f_{5}$, and the curve $f_{1}$. These are shown for comparative purposes.

\subsection{The distribution of cell shapes}

Addressing shape issues when the cells can take any polyhedral form is immensely difficult, because the space of shapes for general polyhedra is extraordinarily complicated (see [8] and [15]). In the $\mathbb{R}^{2}$ case where a domain is triangular (see [7], [9], [10], [11], and [16]) or rectangular (see [2], [5], and [11]), the shape space is more easily managed-as it also is for rectangular prisms in $\mathbb{R}^{d}$ (see [3]).

We focus here on the rectangular case in $\mathbb{R}^{2}$, where the equilibrium distribution of shape in our iteratively dividing models is the most amenable to analysis. Following [2] and [5], we define the shape of a rectangle as the ratio of the shorter side to the longer side, a number in the range $(0,1]$.

Consider the process illustrated in Figure 7(a): area-weighted selection with the dividing chord uniformly chosen from those orthogonal to the longer side of the rectangle. We let $F_{t}(a, \sigma):=\mathrm{P}\{$ a randomly chosen cell at time $t$ has area less than or equal to $a$ and shape less than or equal to $\sigma$, where $0<a \leq t+1$ and $0<\sigma \leq 1$. We define $f_{t}$ as the joint density, if this exists.

It is easier to understand the technique if we derive our equation in the density form, but unfortunately the density does not exist for any finite $t$ in this model, which starts with the $1 \times 1$ square. At time 0 , all probability is concentrated on $(a, \sigma)=(1,1)$. At $t=1$, the randomly chosen cell will have area equal to twice its shape. At $t>1$, there is positive probability mass on the line $a=(t+1) \sigma$, because there is a chance that one of the two cells born at time 1 has not divided again by time $t$. If this occurs then this cell's area is equal to $(t+1)$ times its shape.

To sidestep this problem, we modify the selection of the cell to be divided at $t=3$; we force the choice of the cell whose sister divided at $t=2$. So all four cells at $t=3$ will be second generation, with identical joint probability density function, $f_{3}(a, \sigma)=1 / 4 \sigma, 0<a \leq 4 \sigma$, $0<\sigma \leq 1$. Then we let the process evolve according to the rules of Figure 7(a). The limiting distribution is not dependent on the state at time $t=3$; so our modified process has the same limiting distribution as the original Figure 7(a) process.

Furthermore, following the logic and symbolism which led to (6), but now adapted to be bivariate and in density form, we obtain the following equation. For $t>3$,

$$
\begin{aligned}
f_{t}(a, \sigma) & =\frac{1}{t+1}\left[2 \ell_{t}(a, \sigma)+(t-1) \frac{t}{t+1} \bar{s}_{t-1}\left(\frac{t a}{t+1}, \sigma\right)\right] \\
& =\frac{1}{t+1}\left[2 \ell_{t}(a, \sigma)+\frac{t}{t+1}\left[t f_{t-1}\left(\frac{t a}{t+1}, \sigma\right)-s_{t-1}\left(\frac{t a}{t+1}, \sigma\right)\right]\right],
\end{aligned}
$$

using $\bar{s}_{t-1}=\left[t f_{t-1}-s_{t-1}\right] /(t-1)$. Due to the area-biased selection and the dilation which 
conserves the mean area as 1 , we know that $s_{t}(a, \sigma)=a f_{t}(a, \sigma)$. Also, we can derive $\ell_{t}$ in terms of $s_{t-1}$, as follows:

$$
\begin{aligned}
\ell_{t}(a, \sigma)=\frac{t}{t+1}[ & \int_{\sqrt{a / \sigma(t+1)}}^{1} s_{t-1}\left(\frac{t a}{(t+1) z}, \sigma z\right) \mathrm{d} z \\
& \left.+\frac{1}{\sigma^{2}} \int_{\sqrt{a \sigma /(t+1)}}^{\sigma} s_{t-1}\left(\frac{t a}{(t+1) z}, \frac{z}{\sigma}\right) \mathrm{d} z\right] \\
=\frac{t^{2} a}{(t+1)^{2}} & {\left[\int_{\sqrt{a / \sigma(t+1)}}^{1} f_{t-1}\left(\frac{t a}{(t+1) z}, \sigma z\right) \frac{\mathrm{d} z}{z}\right.} \\
& \left.+\frac{1}{\sigma^{2}} \int_{\sqrt{a \sigma /(t+1)}}^{\sigma} f_{t-1}\left(\frac{t a}{(t+1) z}, \frac{z}{\sigma}\right) \frac{\mathrm{d} z}{z}\right] .
\end{aligned}
$$

Combining (10) and (11) gives a recurrence relationship in $f_{t}$. For $t>3,0<a \leq(t+1) \sigma$, and $0<\sigma \leq 1$,

$$
\begin{aligned}
f_{t}(a, \sigma)= & \frac{2 t^{2} a}{(t+1)^{3}}\left[\int_{\sqrt{a / \sigma(t+1)}}^{1} f_{t-1}\left(\frac{t a}{(t+1) z}, \sigma z\right) \frac{\mathrm{d} z}{z}\right. \\
& \left.+\frac{1}{\sigma^{2}} \int_{\sqrt{a \sigma /(t+1)}}^{\sigma} f_{t-1}\left(\frac{t a}{(t+1) z}, \frac{z}{\sigma}\right) \frac{\mathrm{d} z}{z}\right] \\
& +\frac{t^{2}}{(t+1)^{2}}\left(1-\frac{a}{t+1}\right) f_{t-1}\left(\frac{t a}{t+1}, \sigma\right),
\end{aligned}
$$

whilst $f_{3}(a, \sigma)=1 / 4 \sigma, 0<a \leq 4 \sigma, 0<\sigma \leq 1$.

A MATHEMATICA ${ }^{\circledR}$ routine was used to calculate from (12) the sequence $f_{t}$ algebraically for $t \leq 20$, integrating out with respect to $a$ to get the marginal density of shape (denoted by $h_{t}$ say) for each $t$. For example, $h_{4}(\sigma)=\frac{2}{15}(8-\sigma), h_{5}(\sigma)=\frac{1}{135}\left(149-34 \sigma+9 \sigma^{2}\right)$, and so on, always a polynomial of degree $t-3$. Although the limiting density has not yet been found, the plot in Figure 10 gives an indication of how the shape density behaves as $t$ increases.

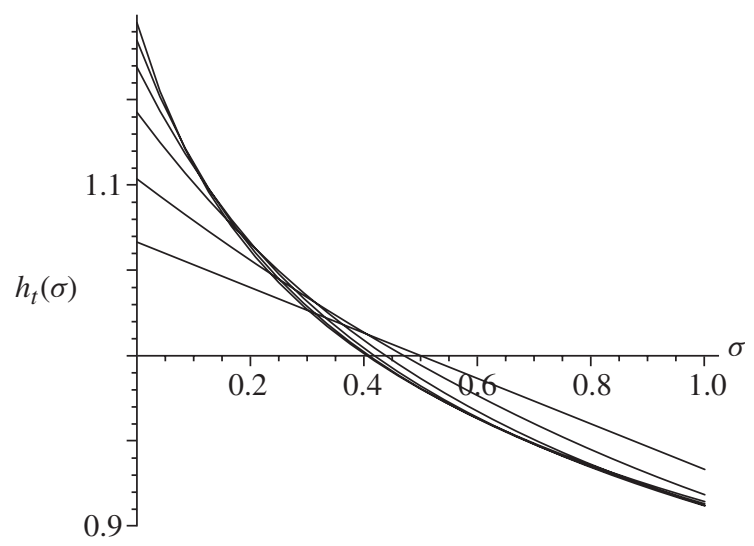

FIGURE 10: The probability density function $h_{t}$ of rectangular cell shape $\sigma$ plotted for $t=4,5,7$, $10,14,20$. The curves can be identified by the fact that $h_{t}(0)$ increases with $t$. Note that, since $h_{t}$ lies in the range $[0.9,1.2]$ for those values of $t$ used, we show the plot only in this range (allowing all curves to be seen more clearly). 
We note that the densities shown are not uniform, but curiously a result in [3] shows that the shape of a cell, with one corner that is also a corner of $W_{t}$, converges to the uniform distribution on $(0,1]$ as $t \rightarrow \infty$; see also [2]. Indeed, if we randomly sample from the $2^{n}$ cells of generation $n$ that are eventually seen as the iterative process evolves, the shape $\sigma_{n}$ which is observed converges to the $\operatorname{Unif}(0,1]$ law as $n \rightarrow \infty$. (See Appendix A for the formal definition of 'generation'.) There are subtle differences between this generationally sampled cell and the cell whose sampling is time based.

\subsection{Convergence of tessellations}

The author has not yet converted the intuitive notions of tessellation convergence presented in this paper into formal theorems. Further work will proceed on this, aiming to characterise precisely the conditions for a limiting locally finite stationary tessellation to exist. We have seen examples where the limiting structure has degenerate cell volume distributions, suggesting strongly that the limit in those examples is not locally finite.

The finite tessellations (in $W_{1}, W_{2}, \ldots$ ) are not stationary, so the methods to achieve this characterisation will not follow exactly those used by Nagel and Weiss in their STIT studies, where each member of the sequence is stationary. It is anticipated however that their methods will readily convert into appropriate theorems for our perimeter-weighted UR chord models.

In general, we anticipate that the convergence of our 'pictorially well-behaved' sequences of tessellations will be established by consideration of the sequence of random closed sets (RACS) formed by the tessellations' frames; see [13] and [14].

\section{Appendix A. Proofs of Theorems 1 and 2}

Cells live for random times. The first cell is born at time $t=0$, whilst a later cell is born when its mother divides. A cell dies at its own epoch of division. When the equally-likely selection rule is used, a cell which is alive at time $s$ will still be alive at time $t>s$ if it is not selected for division at times $(s+1),(s+2),(s+3), \ldots, t$. This has probability

$$
\frac{s}{s+1} \frac{s+1}{s+2} \frac{s+2}{s+3} \cdots \frac{t-1}{t}=\frac{s}{t}
$$

This probability applies to each of the two cells which are born at time $s$, so the expected number of cells born at $s$ who are still alive at $t$ is $2 s / t$. Therefore, the probability that a randomly chosen cell, from the $(t+1)$ cells alive at time $t>0$, was born at time $s \leq t$ is $2 s / t(t+1)$.

The ancestor cell is of generation 0 , whilst a later cell is said to be of generation $n$ if and only if its mother was of generation $n-1$. Let $p_{n}(t)$ denote the probability that a randomly chosen cell at time $t$ belongs to generation $n \leq t$. Clearly, $p_{0}(0)=1$ and $p_{0}(t)=0, t>0$. Also, for $t>0$,

$$
p_{n}(t)= \begin{cases}\frac{2}{t(t+1)}, & n=1, \\ \frac{2}{t(t+1)} \sum_{s=n}^{t} s p_{n-1}(s-1), & n \geq 2 .\end{cases}
$$



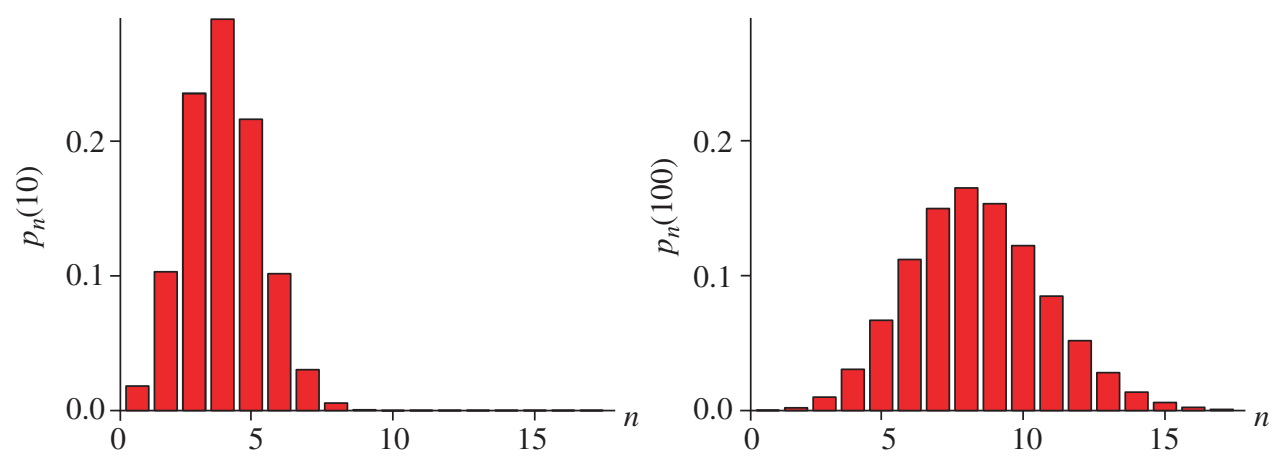

FIGURE 11: The probability mass function $p_{n}(t)$ for the generation number of a randomly sampled cell at time $t$. The two cases are $t=10$ and $t=100$, which have mean values 4.04 and 8.39 , respectively, computed from the exact formula (15).

This can be written, when $n \geq 2$, as

$$
\begin{aligned}
p_{n}(t) & =\frac{2}{t(t+1)}\left[t p_{n-1}(t-1)+\sum_{s=n}^{t-1} s p_{n-1}(s-1)\right] \\
& =\frac{2}{t+1} p_{n-1}(t-1)+\frac{t-1}{t+1} p_{n}(t-1) .
\end{aligned}
$$

Standard methods for the solution of difference equations reveals that $p_{n}(t)$ is the coefficient of $z^{n}$ in the power series $2 z \Gamma(2 z+t) /(t+1) ! \Gamma(2 z+1)$, which equals $(2 z / t(t+1)) \prod_{i=1}^{t-1}(1+$ $2 z / i$ ) when $t>1$. From this probability generating function we can easily find the first two moments of a random variable $N_{t}$ having this distribution:

$$
\begin{gathered}
\mathrm{E} N_{t}=2\left(\sum_{j=1}^{t+1} \frac{1}{j}-1\right) \approx 2(\log (t+1)+\gamma-1), \\
\operatorname{var} N_{t}=2\left(3+\sum_{j=1}^{t+1} \frac{1}{j}-2 \sum_{j=1}^{t+1} \frac{1}{j^{2}}\right) \approx 2\left(1+2 \log (t+1)+2 \gamma-\frac{\pi^{2}}{3}\right) .
\end{gathered}
$$

The approximations are for large $t$. Here $\gamma$ is Euler's constant. Figure 11 shows the variation of $N_{t}$, described by $p_{n}(t)$.

Proof of Theorem 1. We note that all cells of generation $n$ are the result of $n$ divisions. Initially, consider these divisions in the absence of dilation. The first division (at time $t=1$ ) creates daughter cells each with volume distribution $G_{1}$. The second creates daughters with distribution $G_{2}$, the third $G_{3}$, and so on. So, in a process where dilation is absent, the volume distribution at time $t$ is a mixture, $\sum_{1 \leq n \leq t} p_{n}(t) G_{n}(x)$ for $0 \leq x \leq 1$.

Now adjusting for the dilation (which can be achieved by one single dilation of $W_{0}$ to create $W_{t}$ whose volume is $(t+1)$, instead of progressive dilations at each division epoch), result (3) in Theorem 1 follows:

$$
F_{t}(x)=\sum_{n=1}^{t} p_{n}(t) G_{n}\left(\frac{x}{t+1}\right) .
$$

A proof of Theorem 1 can also be achieved by the formal substitution of (3) into (2). The 
right-hand side is

$$
\begin{aligned}
F_{t-1}( & \left.\frac{t x}{t+1}\right)+\frac{2}{t+1} \int_{v=t x /(t+1)}^{v=t} G\left(\frac{t x}{(t+1) v}\right) \mathrm{d} F_{t-1}(v) \\
= & \sum_{n=1}^{t-1} p_{n}(t-1) G_{n}\left(\frac{x}{t+1}\right)+\frac{2}{t+1} \int_{v=t x /(t+1)}^{v=t} G\left(\frac{t x}{(t+1) v}\right) \sum_{j=1}^{t-1} p_{j}(t-1) \mathrm{d} G_{j}\left(\frac{v}{t}\right) \\
= & \sum_{n=1}^{t-1} p_{n}(t-1) G_{n}\left(\frac{x}{t+1}\right) \\
& \left.+\frac{2}{t+1} \sum_{j=1}^{t-1} p_{j}(t-1) \int_{u=x /(t+1)}^{u} G\left(\frac{x}{(t+1) u}\right) \mathrm{d} G_{j}(u) \quad \text { (where } u=v / t\right) \\
= & \sum_{n=1}^{t-1} p_{n}(t-1) G_{n}\left(\frac{x}{t+1}\right) \\
& +\frac{2}{t+1} \sum_{j=1}^{t-1} p_{j}(t-1)\left[G_{j+1}\left(\frac{x}{t+1}\right)-G_{j}\left(\frac{x}{t+1}\right)\right] \quad(\text { from }(4)) \\
= & \frac{t-1}{t+1} \sum_{n=1}^{t-1} p_{n}(t-1) G_{n}\left(\frac{x}{t+1}\right)+\frac{2}{t+1} \sum_{n=2}^{t} p_{n-1}(t-1) G_{n}\left(\frac{x}{t+1}\right) \\
= & \frac{t-1}{t+1} p_{1}(t-1) G_{1}\left(\frac{x}{t+1}\right) \\
& +\frac{1}{t+1} \sum_{n=2}^{t-1}\left[2 p_{n-1}(t-1)+(t-1) p_{n}(t-1)\right] G_{n}\left(\frac{x}{t+1}\right) \\
& \sum_{n=1}^{t} p_{t-1}(t) G_{t}\left(\frac{x}{t+1}\right) \\
& \left.+\frac{x}{t+1}\right)=F_{t}(x) \quad(\mathrm{using}(13) \text { and }(14)) . \\
& \\
& \\
&
\end{aligned}
$$

The proof that (3) is a solution of (2) is now complete (by two different arguments). The uniqueness of this solution is clear; once $G$ and $F_{0}$ are given, (2) is a function mapping to just one member $F_{1}$ in the space of distribution functions. Similarly, once $F_{1}$ is established, $F_{2}$ is specified by a functional mapping, and so on.

Proof of Corollary 1. A typical cell with length $V_{t}$ and belonging to generation $N_{t}$ is sampled. Obviously, $\mathrm{E}\left(V_{t}^{2} \mid N_{t}=n\right)=(t+1)^{2}\left(\mathrm{E} U^{2}\right)^{n}$; so $\mathrm{E}\left(V_{t}\right)=(t+1)^{2} \sum_{n=1}^{t} p_{n}(t)\left(\mathrm{E} U^{2}\right)^{n}$. The variance follows using $\mathrm{E} V_{t}=1$. The infinite limit as $t \rightarrow \infty$ follows because $\mathrm{E} U^{2} \leq 1$ and $\sum_{n} p_{n}(t)=1$. Note also that $\mathrm{E} U^{2} \geq \frac{1}{4}$, the lower bound being achieved when $\mathrm{P}\left\{U=\frac{1}{2}\right\}=1$.

Proof of Theorem 2. We aim to show that $F_{t}(x)$ given in (17) tends to 1 as $t \rightarrow \infty$ for all $x>0$ - which implies the same result for $x=0$, due to the right continuity of $F_{t}$.

Recall that $G_{n}(x)=0$ when $x<1 / 2^{n}$ and $G_{n}(x)=1$ otherwise. Take a particular fixed $x>0$. Define $n(t):=\max \left(n: x /(t+1)<1 / 2^{n}\right)$. That is, $n(t)=[(\log (t+1)-\log (x)) / \log (2)]$, where [.] denotes the integer part. From (17), $F_{t}(x)=\sum_{n>n(t)} p_{n}(t)=1-\sum_{1 \leq n \leq n(t)} p_{n}(t)$. 
We now show that $\sum_{1 \leq n \leq n(t)} p_{n}(t)$, which equals $\mathrm{P}\left\{N_{t} \leq n(t)\right\}$, tends to 0 , using Chebyshev's inequality. This inequality tells us that, for $a>0$,

$$
\mathrm{P}\left\{\left|N_{t}-\mathrm{E} N_{t}\right| \geq a\right\} \leq \frac{\operatorname{var} N_{t}}{a^{2}} .
$$

Therefore, setting $a=\mathrm{E} N_{t}-n(t)$, we obtain

$$
\begin{aligned}
\mathrm{P}\left\{N_{t} \leq n(t)\right\} & =\mathrm{P}\left\{N_{t}-\mathrm{E} N_{t} \leq n(t)-\mathrm{E} N_{t}\right\} \\
& \leq \mathrm{P}\left\{N_{t}-\mathrm{E} N_{t} \leq n(t)-\mathrm{E} N_{t}\right\}+\mathrm{P}\left\{N_{t}-\mathrm{E} N_{t} \geq \mathrm{E} N_{t}-n(t)\right\} \\
& =\mathrm{P}\left\{\left|N_{t}-\mathrm{E} N_{t}\right| \geq \mathrm{E} N_{t}-n(t)\right\} \\
& \leq \frac{\operatorname{var} N_{t}}{\left(\mathrm{E} N_{t}-n(t)\right)^{2}},
\end{aligned}
$$

provided that $\mathrm{E} N_{t}-n(t)>0$. So, if there exists a number $t_{x}$ such that $\mathrm{E} N_{t}-n(t)>0$ for all $t>t_{x}$, then (18) will provide the proof we need (see below).

To find firstly if $t_{x}$ exists, we note that

$$
\begin{gathered}
n(t)=\left[\frac{\log (t+1)-\log (x)}{\log 2}\right] \leq \frac{\log (t+1)-\log (x)}{\log 2}=B_{1}(t) \quad \text { (say) } \\
\text { and } \mathrm{E} N_{t}>2(\log (t+1)+\gamma-1)=B_{2}(t) .
\end{gathered}
$$

The inequality in (20) follows from the equality in (15) because $c_{t}$, defined as $\sum_{j=1}^{t+1} 1 / j-$ $\log (t+1)$, is a monotone decreasing sequence (as confirmed by $c_{t+1}-c_{t}=1 /(t+2)+$ $\log [1-1 /(t+2)]<0)$. So $c_{t}>\lim _{n \rightarrow \infty} c_{n}=\gamma$.

Elementary algebra shows that the lower bound $B_{2}(t)$ in (20) is greater than the upper bound $B_{1}(t)$ in (19), implying that $\mathrm{E} N_{t}>n(t)$, if

$$
t>\left(\frac{4^{(1-\gamma)}}{x}\right)^{1 /(\log 4-1)}-1 \approx \frac{4.56}{x^{2.59}}-1 .
$$

This lower bound for $t$ plays the role of $t_{x}$.

Returning to (18), now known to be valid for $t>t_{x}$,

$$
\mathrm{P}\left\{N_{t} \leq n(t)\right\} \leq \frac{\operatorname{var} N_{t}}{\left(\mathrm{E} N_{t}-n(t)\right)^{2}}<\frac{\operatorname{var} N_{t}}{\left(B_{2}(t)-B_{1}(t)\right)^{2}} .
$$

Note that var $N_{t} \sim \log (t+1)$ and the denominator grows like $(\log (t+1))^{2}$, because we can easily show that $B_{2}(t)-B_{1}(t) \sim \log (t+1)$. Therefore, the bound in (21) tends to 0 as $t \rightarrow \infty$.

So the proof of Theorem 2 is complete.

\section{Acknowledgement}

I thank the anonymous referees for their comments on an earlier version of this paper.

\section{References}

[1] Abramowitz, M. and Stegun, I. A. (1970). Handbook of Mathematical Functions. Dover, New York.

[2] Chen, F. K. C. AND Cowan, R. (1999). Invariant distributions for shapes in sequences of randomly-divided rectangles. Adv. Appl. Prob. 31, 1-14.

[3] Cowan, R. (1997). Shapes of rectangular prisms after repeated random division. Adv. Appl. Prob. 29, 26-37. 
[4] Cowan, R. (2004). A mosaic of triangular cells formed with sequential splitting rules. In Stochastic Methods and Their Applications (J. Appl. Prob. Spec. Vol. 41A), eds J. Gani and E. Seneta, Applied Probability Trust, Sheffield, pp. 3-15.

[5] Cowan, R. And Chen, F. K. C. (1999). Four interesting problems concerning Markovian shape sequences. Adv. Appl. Prob. 31, 954-968.

[6] Halmos, P. R. (1944). Random alms. Ann. Math. Statist. 15, 182-189.

[7] Kendall, D. G. (1977). The diffusion of shape. Adv. Appl. Prob. 9, 428-430.

[8] Kendall, D. G., Barden, D., Carne, T. K. and Le, H. (1999). Shape and Shape Theory. John Wiley, Chichester.

[9] Mannion, D. (1990). Convergence to collinearity of a sequence of random triangle shapes. Adv. Appl. Prob. 22, 831-844.

[10] Mannion, D. (1993). Products of $2 \times 2$ random matrices. Ann. Appl. Prob. 3, 1189-1218.

[11] Miles, R. E. (1983). On the repeated splitting of a planar domain. In Proc. Oberwolfach Conf. Stochastic Geometry, Geometric Statistics and Stereology, eds R. Ambartzumian and W. Weil Teubner, Leipzig, pp. 110-123.

[12] Mecke, J., Nagel, W, And Weiss, V. (2007). Length distributions of edges in planar stationary and isotropic STIT tessellations. Izv. Akad. Nauk Armenii Mat. 42, 39-60.

[13] Nagel, W. AND WeIss, V. (2005). Crack STIT tessellations: characterization of stationary random tessellations stable with respect to iteration. Adv. Appl. Prob. 37, 859-883.

[14] Norberg, T. (1984). Convergence and existence of random set distributions. Ann. Prob. 12, 726-732.

[15] Small, C. G. (1996). The Statistical Theory of Shape. Springer, New York.

[16] Watson, G. S. (1986). The shapes of a random sequence of triangles. Adv. Appl. Prob. 18, 156-169. 